\title{
Enhanced adhesion and proliferation of bone marrow mesenchymal stem cells on $\beta$-tricalcium phosphate modified by an affinity peptide
}

\author{
GUOZONG WANG ${ }^{1,2}$, ZHENTAO MAN $^{1}$, HUA XIN $^{3}$, YI LI $^{1}$, CHANGSHUN WU $^{1}$ and SHUI SUN ${ }^{1}$ \\ ${ }^{1}$ Department of Joint Surgery, Shandong Provincial Hospital Affiliated to Shandong University, Jinan, \\ Shandong 250021; ${ }^{2}$ College of Clinical Medicine, Shandong University, Jinan, Shandong 250012; \\ ${ }^{3}$ Department of Neurology, People's Hospital of Rizhao, Rizhao, Shandong 222000, P.R. China
}

Received April 26, 2018; Accepted October 9, 2018

DOI: $10.3892 / \mathrm{mmr} .2018 .9655$

\begin{abstract}
Mesenchymal stem cells (MSCs) are often used in orthopedic tissue engineering, and bone marrow-derived mesenchymal stem cells (BMSCs) are currently considered the gold standard. One of the most important issues in MSC-based tissue engineering therapy is the low number of MSCs in pathological tissues. Achieving efficient recruitment of MSCs to defective or damaged tissues in vivo has been a difficult hurdle. The aim of the present study was to construct a biomaterial that can effectively recruit BMSCs to facilitate the repair of pathological tissues. So functional $\beta$-tricalcium phosphate $(\beta$-TCP) was synthesized using the BMSC affinity peptide DPIYALSWSGMA (DPI) adsorbed onto $\beta$-TCP through an adsorption/freeze-drying strategy. C57BL/6 mouse-derived BMSCs were seeded onto the DPI peptide-modified $\beta$-TCP ( $\beta$-TCP-DPI); in vitro experiments demonstrated that $\beta$-TCP-DPI enhanced BMSC adhesion and proliferation compared with unmodified $\beta$-TCP. Results from the present study indicated that functional $\beta$-TCP may be used as an ideal scaffold in tissue engineering and in regenerative medicine.
\end{abstract}

\section{Introduction}

Refractory diseases, including osteonecrosis of the femoral head, are a growing worldwide health problem (1), and the potential of tissue engineering strategies for their treatment are currently being investigated. The fundamental elements of

Correspondence to: Professor Shui Sun, Department of Joint Surgery, Shandong Provincial Hospital Affiliated to Shandong University, 324 Jingwuweiqi Road, Huaiyin, Jinan, Shandong 250021, P.R. China

E-mail: sunshui1965@foxmail.com

Key words: orthopedic tissue engineering, bone marrow mesenchymal stem cells, $\beta$-tricalcium phosphate, affinity peptide, adhesion, proliferation tissue engineering include scaffolds, signals and cells. Tissue engineering scaffolds are cytocompatible biomaterials that cells adhere to and/or replace with extracellular matrix (ECM) to produce native tissues (2). Scaffolds are usually classified on the basis of their source and ability to degrade. Tricalcium phosphate (TCP) is a synthetic, degradable inorganic material that has good biocompatibility, bioactivity and biodegradability; it is an ideal tissue repair material (3). TCP is commonly used at low temperature $\beta$ phase, and $\beta$-TCP is mainly composed of calcium and phosphorus, similar to the inorganic composition of bone (4). However, it is a challenging material owing to its hydrophobicity and the absence of active groups to interact with cells of interest.

Mesenchymal stem cells (MSCs) are the most common type of cell used in orthopedic tissue engineering. MSCs have the potential to differentiate into osteoblasts, chondrocytes and adipocytes (5). At present, bone marrow-derived MSCs (BMSCs) are considered the gold standard for use in tissue engineering (2). However, pathological tissues often have poor innate regenerative capacity, with few or no viable MSCs (2). In tissue engineering and regenerative medicine, seed cells (for example, BMSCs) should be combined with scaffolds to repair tissues of interest (6). Strategies for taking full advantage of biomaterials and the efficient recruitment of MSCs are being increasingly investigated (7). The precise and efficient adhesion of MSCs to biomaterials requires investigation.

A number of attempts to enhance the affinity between cells and biomaterials have been conducted, and surface modification of biomaterials is widely used. For example, the heptapeptide sequence, LTHPRWP (L7), with affinity towards synovium-derived mesenchymal stem cells (SMSCs) has been covalently conjugated to polycaprolactone electrospun meshes and to human decalcified bone scaffolds; this elevates the adhesion and spreading of SMSCs on scaffolds (8). A chondrocyte-affinity peptide (CAP), DWRVIIPPRPSA, was identified by phage display technology (9). Polyethylenimine has been covalently modified with CAP to construct a non-viral vector for cartilage-targeted therapy (9). A previous study revealed the enhanced adhesion of human dermal fibroblasts on anorganic bovine bone mineral modified by the functional synthetic 15-residue peptide sequence GTPGPQGIAGQRQVV (P-15), 
which is a potent cell-binding domain in the $\alpha 1$ chain of type I collagen $(10,11)$. The RGD peptide, derived from fibronectin in ECM, was demonstrated to promote cell adhesion in 1984 (12); since then, a number of materials modified with RGD have been used in academic study and clinical therapies $(13,14)$.

Affinity peptides towards BMSCs are also commonly used. Recently, a novel peptide, DPIYALSWSGMA (DPI), with specific affinity towards BMSCs, was identified through phage display technology (15). In the present study, the affinity of the DPI peptide towards BMSCs was further verified. $\beta$-TCP was selected as the scaffold modified by DPI and cellular behavior on the modified scaffold was studied.

\section{Materials and methods}

Cell culture.C57BL/6 mouse BMSCs (cat.no.MUBMX-01001) were obtained from Cyagen Biosciences, Inc. (Santa Clara, CA, USA). The cells were cultured in cell culture flasks in a humidified atmosphere with $5 \% \mathrm{CO}_{2}$ at $37^{\circ} \mathrm{C}$ (Fig. 1). Cells were cultured in low glucose Dulbecco's modified Eagle's medium (cat. no. 01-051-1A; Biological Industries, Kibbutz Beit-Haemek, Israel) with L-glutamine containing $10 \%$ fetal bovine serum (cat. no. 10099141; Gibco; Thermo Fisher Scientific, Inc., Waltham, MA, USA) and antibiotics $(100 \mathrm{U} / \mathrm{ml}$ penicillin and $0.1 \mathrm{mg} / \mathrm{ml}$ streptomycin; cat. no. 15140122; Gibco; Thermo Fisher Scientific, Inc.). The medium was replaced every 2-3 days. Cells were detached and passaged at $80-90 \%$ confluency. BMSCs were used at passage 4-5 for further experiments.

Peptide synthesis. The DPI peptide sequence with high affinity towards BMSCs (Fig. 2A) was previously discovered using phage display (15). The linear dodecapeptide was derived from the Ph.D.-12 phage display library (cat. no. E8110S; New England Biolabs, Inc., Ipswich, MA, USA). The molecular weight of the synthesized DPI peptide was confirmed to be 1,311.58 Da by mass spectrometry (JBI Scientific, LLC, Huntsville, TX, USA) using matrix-assisted laser desorption/ionization-time of flight method with positive mode (Fig. 2B). The synthesized DPI peptide was dissolved in $50 \%$ acetonitrile (ACN) with $0.05 \%$ trifluoroacetic acid (TFA) to obtain a concentration of $100 \mu \mathrm{g} / \mathrm{ml}$, and subsequently mixed with $\alpha$-cyano-4-hydroxycinnamic acid solution $(10 \mathrm{mg} / \mathrm{ml}$ in $50 \%$ ACN, $0.05 \%$ TFA) at a 1:1 ratio. A total of $1 \mu \mathrm{l}$ of the mixture was loaded on target plate and air dried. The instrument settings were: $20,000 \mathrm{~V}$ accelerating voltage, $95 \%$ grid voltage, acquisition mass range between 600 and 2,000 Da, and extraction delay time was $200 \mathrm{nsec}$. A peptide sequence of the same chain length as DPI, but scrambled (LSPSAGAYIDWM; LSP), was used as the negative control. A peptide comprising three amino acids (RGD) was used as the positive control. All peptides were synthesized by solid-phase peptide synthesis using 9-fluorenylmethoxycarbonyl chemistry (Scilight-Peptide, Inc.; Scilight Biotechnology, LLC Beijing, China). An extra aminohexanoic acid was linked at the amino-terminus of all peptides to facilitate fluorescein-5-isothiocyanate (FITC) labeling. The FITC-labeled peptides, FITC-DPI, FITC-LSP and FITC-RGD, were stored at $-20^{\circ} \mathrm{C}$. A concentration of $1 \mathrm{mg} / \mathrm{ml}$ was obtained by dissolving the peptides in PBS (cat. no. 02-024-1A; Biological Industries) before use.
Peptide-affinity assay by flow cytometry. The C57BL/6 mouse BMSCs were washed twice with PBS and dissociated with $0.25 \%$ trypsin-EDTA (cat. no. 25200-056; Gibco; Thermo Fisher Scientific, Inc.). The cell suspension was centrifuged at $250 \mathrm{x}$ g for $5 \mathrm{~min}$ at room temperature to collect cell sedimentation. The cells were incubated with $100 \mu \mathrm{M}$ FITC-labeled peptides for $1 \mathrm{~h}$ at $37^{\circ} \mathrm{C}$ to allow cell binding and internalization. The mouse BMSC affinity properties of the peptides were analyzed quantitatively using flow cytometry at a wavelength of $488 \mathrm{~nm}$ and FlowJo v7.6.1 (Tree Star, Inc., Ashland, OR, USA) software. All procedures were repeated at least three times.

Peptide-affinity assay by fluorescence cytochemistry. C57BL/6 mouse BMSCs were cultured in 24-well dishes until $70-90 \%$ confluence was achieved. The cells were subsequently incubated with $100 \mu \mathrm{M}$ FITC-labeled peptides for $1 \mathrm{~h}$ at $37^{\circ} \mathrm{C}$ and with rhodamine-labeled phalloidin (cat. no. CA1610; Beijing Solarbio Science \& Technology Co., Ltd., Beijing, China) for $30 \mathrm{~min}$ at room temperature for cytoskeletal staining. The nuclei were counterstained with DAPI (cat. no. C0065; Beijing Solarbio Science \& Technology Co., Ltd.). The cells were examined in the 24-well dishes using a fluorescence microscope. All procedures were repeated at least three times.

Synthesis of DPI-modified $\beta$-TCP $(\beta-T C P$-DPI $)$. The synthesis of $\beta$-TCP-DPI was conducted following a previously described procedure $(10,16)$. Functional $\beta$-TCP scaffolds were constructed using DPI peptides that were adsorbed onto $\beta$-TCP through an adsorption/freeze-drying strategy. Briefly, disk-shaped $\beta$-TCP (diameter, 6 mm; height, 2 mm; Shanghai Bio-lu Biomaterials Co., Ltd, Shanghai, China) was incubated for $24 \mathrm{~h}$ at room temperature in peptide solution containing $100 \mu \mathrm{g} / \mathrm{ml}$ DPI in PBS in a ratio of $1.0 \mathrm{~g} \beta$-TCP to $2.0 \mathrm{ml}$ solution with gentle agitation to ensure to equilibrate the peptide over all exposed surfaces of the microporous $\beta$-TCP. Unadsorbed peptide was removed from the scaffolds by washing five times in PBS with gentle shaking over a $24 \mathrm{~h}$ period. The $\beta$-TCP-DPI composites were dried in vacuo for $1 \mathrm{~h}$ and stored at $-20^{\circ} \mathrm{C}$ in moisture-proof containers. FITC-DPI peptide-modified $\beta$-TCP was also synthesized and observed using ImageXpress Micro Confocal (Molecular Devices, LLC, Sunnyvale, CA, USA). The $\beta$-TCP and $\beta$-TCP-DPI composites were sterilized under ultraviolet (UV) light for cell culture experiments.

Behavior of cells on $\beta$-TCP-DPI in vitro. C57BL/6 mouse BMSCs were seeded at passage 4-5 onto pure $\beta$-TCP and $\beta$-TCP-DPI scaffolds to investigate cell adhesion and proliferation, as described below. All scaffolds were sterilized through UV light exposure on a clean bench before use.

Cell adhesion assay. Cell Counting Kit-8 (CCK-8; cat. no., 96992; Sigma-Aldrich; Merck KGaA, Darmstadt, Germany) was used to evaluate the number of cells adhered onto the scaffolds (17). The cells were dissociated with $0.25 \%$ trypsin-EDTA. The cell suspension was centrifuged at $250 \mathrm{x} g$ for $5 \mathrm{~min}$ at room temperature and the sediment was collected. Cells were resuspended at a specified concentration $\left(6 \times 10^{4}\right.$ cells $\left./ \mathrm{ml}\right)$ and an appropriate volume $(200 \mu \mathrm{l})$ in serum-free DMEM. The cell suspension was added to 96 -well plates containing $\beta$-TCP and $\beta$-TCP-DPI and incubated for $3 \mathrm{~h}$ 

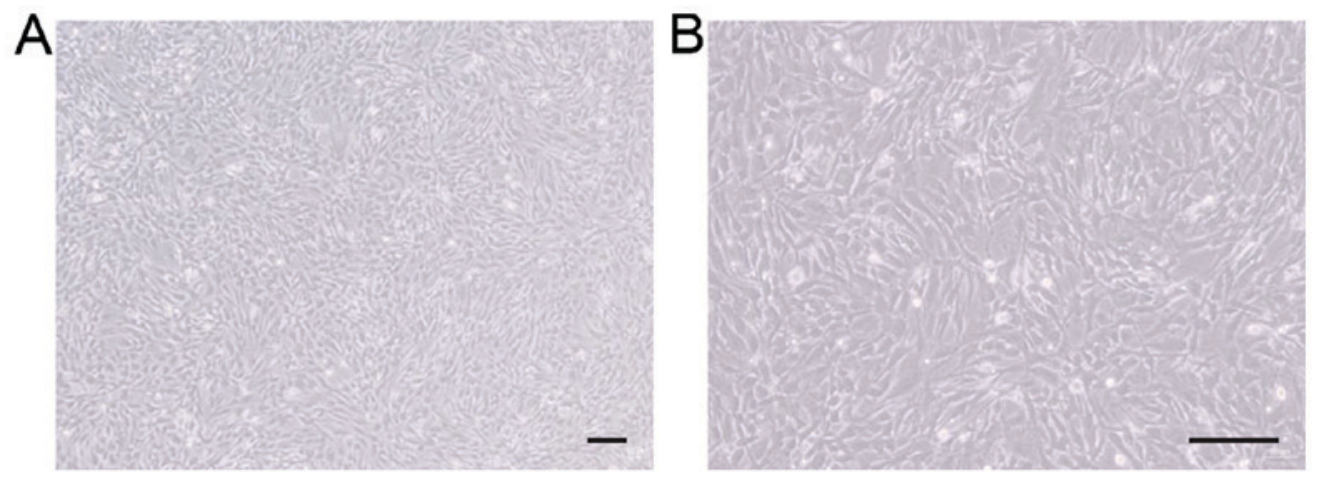

Figure 1. C57BL/6 mouse BMSCs were cultured to prepare for subsequent experiments. (A and B) At passage 4, mouse BMSCs exhibited a spindle-shaped or fibroblastic appearance; (A) magnification, $\mathrm{x} 40$, and (B) magnification, $\mathrm{x} 100$; scale bar, $250 \mu \mathrm{m}$. BMSC, bone marrow mesenchymal stem cell.

A

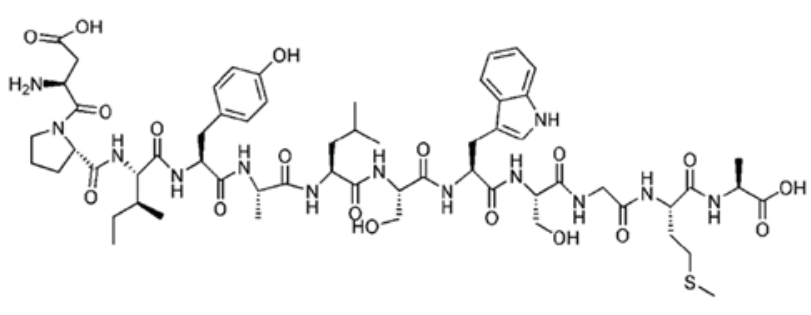

Asp-Pro-Ile-Tyr-Ala-Leu-Ser-Trp-Ser-Gly-Met-Ala
B

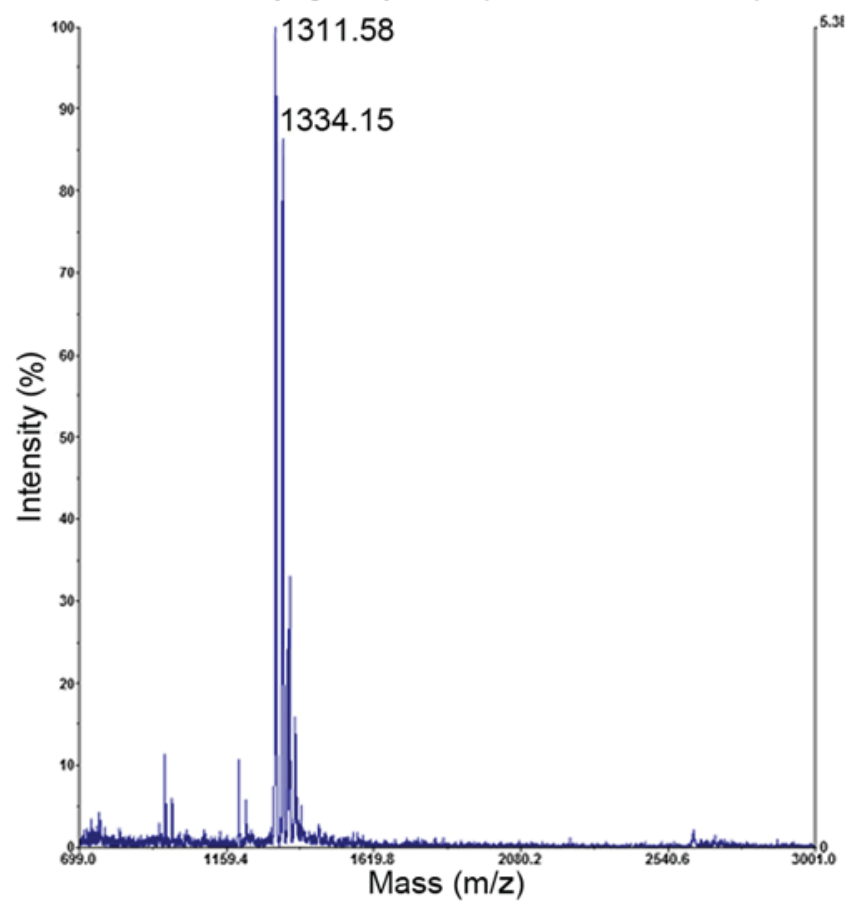

Figure 2. A linear dodecapeptide identified using phage display technology. (A) A peptide sequence, DPI, with a highly specific affinity towards BMSCs was identified by phage display. (B) The molecular weight of the synthesized DPI peptide was confirmed to be 1,311.58 Da by mass spectrometry. BMSC, bone marrow mesenchymal stem cell; DPI, DPIYALSWSGMA.

in a humidified atmosphere with $5 \% \mathrm{CO}_{2}$ at $37^{\circ} \mathrm{C}$; the scaffolds were removed to new wells and washed thrice with PBS. Subsequently, $100 \mu \mathrm{l}$ fresh medium and $10 \mu \mathrm{l}$ CCK- 8 reagent were added and the BMSCs that adhered to the scaffolds were incubated for another $4 \mathrm{~h}$ at $37^{\circ} \mathrm{C}$. The absorbance was measured at $450 \mathrm{~nm}$ using an automated microplate reader. All procedures were repeated at least three times.

Cell proliferation assay. CCK- 8 assay was used to measure proliferation of cells on $\beta$-TCP-DPI and unmodified $\beta$-TCP scaffolds. C57BL/6 mouse BMSCs were seeded onto the unmodified $\beta$-TCP and $\beta$-TCP-DPI scaffolds. Briefly, a total of $30 \mu \mathrm{l}$ cell suspension containing $2 \times 10^{3}$ cells was slowly and carefully pipetted onto the center-top surface of each $\beta$-TCP and $\beta$-TCP-DPI disk. The cell suspension was not allowed to contact the sides of the wells to ensure that all cells adhered to the scaffolds. Plates were gently placed into an incubator. After 3-h incubation, an extra volume (170 $\mu \mathrm{l})$ fresh medium was added to each well gently and slowly along the edge of the well; wells were not rinsed to avoid washing out cells that did not firmly adhere to the scaffolds. After 3 days of incubation, the scaffolds were washed thrice with PBS and incubated with CCK-8 solution at $37^{\circ} \mathrm{C}$ for $3 \mathrm{~h}$. Absorbance was measured at $450 \mathrm{~nm}$ with an automated microplate reader. All procedures were repeated at least three times.

Statistical analysis. Data are expressed as the mean \pm standard deviation. Student's t-test was performed to compare two groups and one-way analysis of variance followed by Dunnett's test was performed for comparison of multiple groups. SPSS v24.0 


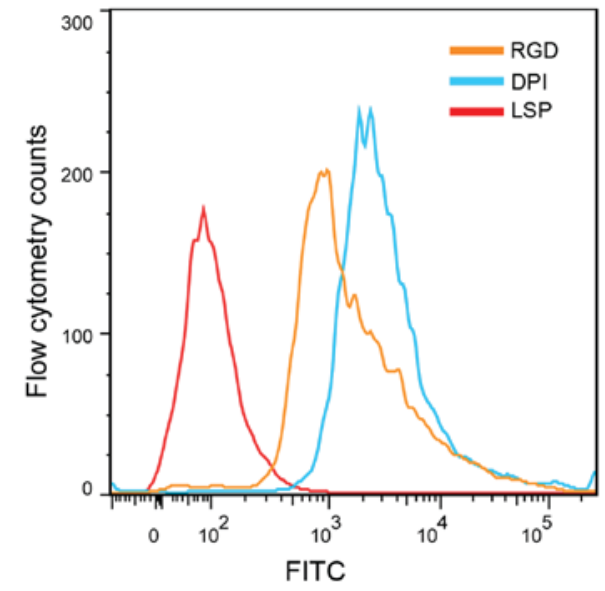

Figure 3. DPI peptide exhibits high affinity towards C57BL/6 mouse BMSCs. C57BL/6 mouse BMSCs were incubated with FITC-labeled DPI, RGD or LSP, and the fluorescence was analyzed by flow cytometry. The peaks of the experimental (DPI) and positive control (RGD) groups shifted to the right compared with the negative control group (LSP), which indicated that DPI had a high affinity for the target cells. BMSC, bone marrow mesenchymal stem cell; DPI, DPIYALSWSGMA; FITC, fluorescein isothiocyanate; LSP,LSPSAGAYIDWM.

(IBM Corp., Armonk, NY, USA) software was used for data analysis. $\mathrm{P}<0.05$ was considered to indicate a statistically significant difference.

\section{Results}

DPI has a highly specific affinity towards mouse BMSCs. The mouse BMSC affinity peptide DPI, the negative control peptide LSP and the positive control peptide RGD were used in this study. Mouse BMSCs were incubated for $1 \mathrm{~h}$ with FITC-labeled DPI, LSP or RGD, and analyzed by flow cytometry. The average fluorescence intensity was $7,343.5 \pm 167.6$ for BMSCs incubated with FITC-DPI, 7,042.0 179.6 for BMSCs incubated with FITC-RGD and 132.0 \pm 80.6 for BMSCs incubated with FITC-LSP. The average fluorescence intensities for BMSCs incubated with FITC-DPI and FITC-RGD were significantly higher compared with BMSCs incubated with FITC-LSP $(\mathrm{n}=3$; $\mathrm{P}<0.01$; Figs. 3 and 4). The average fluorescence intensity of cells incubated with FITC-DPI was 55.6-fold higher than that of FITC-LSP. The cells were also observed under a fluorescence microscope. Strong fluorescent signals were observed in the cells incubated with FITC-DPI and FITC-RGD, whereas weak fluorescent signals were observed of the cells incubated with FITC-LSP (Fig. 5). These results suggested that the DPI peptide may have a high affinity for mouse BMSCs.

Successful synthesis of functional $\beta$-TCP scaffolds. FITC-DPI was adsorbed onto the $\beta$-TCP scaffolds for surface modification through an adsorption/freeze-drying strategy. Following adsorption, the $\beta$-TCP-DPI scaffolds exhibited homogeneous green fluorescence (Fig. 6). This result indicated successful adsorption of DPI onto the surface of $\beta$-TCP scaffolds and the successful construction of $\beta$-TCP-DPI composite materials.

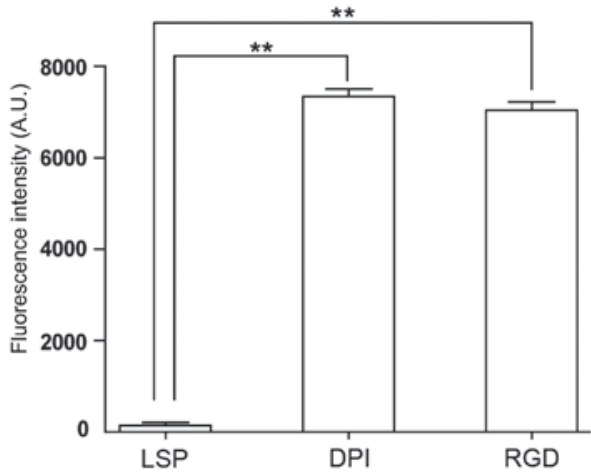

Figure 4. Affinity of DPI towards C57BL/6 mouse BMSCs is verified by flow cytometry. The affinity of DPI peptides towards BMSCs was analyzed quantitatively by flow cytometry following incubation with FITC-labeled LSP, DPI or RGD. The average fluorescence intensity of cells incubated with FITC-DPI and FITC-RGD was significantly higher compared with that of FITC-LSP. Data are expressed as the mean \pm standard deviation; $n=3$; ${ }^{* *} \mathrm{P}<0.01$ vs. LSP. A.U., arbitrary units; BMSC, bone marrow mesenchymal stem cell; DPI, DPIYALSWSGMA; FITC, fluorescein isothiocyanate; LSP, LSPSAGAYIDWM.

scaffolds. In the cell adhesion assay, the number of adherent cells on the $\beta$-TCP-DPI composites was significantly higher compared with those on the pure $\beta$-TCP scaffolds, according to optical density (OD) measurements $(n=3 ; P<0.01$; Fig. 7). This result indicated that the $\beta$-TCP-DPI composites are effective for mouse BMSC adhesion.

A CCK-8 assay was used to evaluate cell proliferation on pure $\beta$-TCP and $\beta$-TCP-DPI scaffolds. Following 3 days of incubation, the OD value of $\beta$-TCP-DPI scaffolds was significantly higher compared with pure $\beta$-TCP scaffolds, which indicated that $\beta$-TCP-DPI may enhance BMSC proliferation compared with unmodified $\beta$-TCP $(n=3 ; P<0.01 ;$ Fig. 8$)$.

\section{Discussion}

Tissue engineering uses concepts of biology and engineering to develop functional substitutes for damaged tissue (18), and MSCs are a common type of seed cell used for tissue engineering. MSCs in human bone marrow have been estimated to comprise $0.001-0.01 \%$ of the total nucleated cells, and this number declines with age (19). Furthermore, MSCs are rare or absent in pathological tissues such as the necrotic femoral head (2). A number of studies have proposed that host progenitor cells build new bone following recruitment to the site of repair $(20,21)$. A method of efficient recruitment and utilization of the limited number of MSCs remains to be established. The strategy of recruiting BMSCs using affinity peptides is commonly adopted $(14,22)$. Phage display provides a novel method for searching of highly specific affinity peptides towards BMSCs. In this way, a number of peptide sequences with highly specific affinity towards BMSCs have been identified and used to modify biomaterials to improve their surface properties and functions. For example, the peptide sequence EPLQLKM (E7) is widely used to enhance the interaction between BMSCs and various scaffolds $(6,23-26)$. In the present study, the DPI peptide, with affinity towards BMSCs, was also reported to be discovered through phage display (15). Flow cytometry and fluorescence cytochemistry were used to confirm the high affinity of DPI 


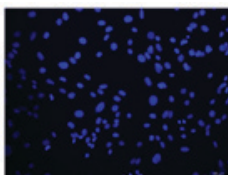

DAPI

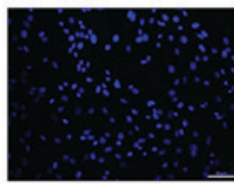

DAPI

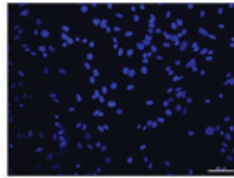

DAPI

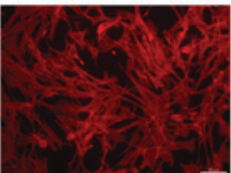

Phalloidin

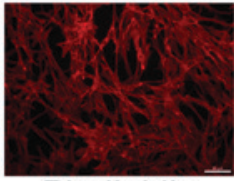

Phalloidin

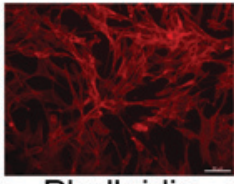

Phalloidin

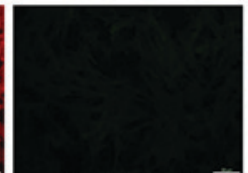

FITC-LSP

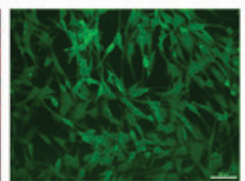

FITC-DPI

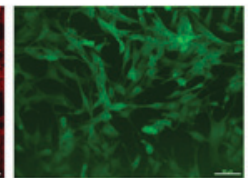

FITC-RGD

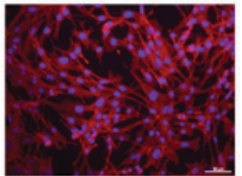

Merge

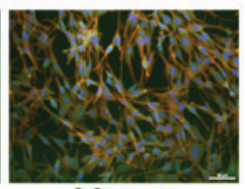

Merge

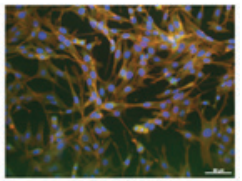

Merge

Figure 5. DPI exhibits high affinity towards C57BL/6 mouse BMSCs. Affinity of the linear dodecapeptide, DPI, towards C57BL/6 mouse BMSCs was investigated through fluorescence staining. Cells were incubated with FITC-labeled DPI, RGD or LSP and observed under a fluorescence microscope. Strong FITC signals were observed in cells incubated with FITC-DPI and FITC-RGD, whereas weak signals were observed of cells incubated with FITC-LSP. Nuclei were stained with DAPI and the cytoskeleton was stained with rhodamine phalloidin; scale bar, $50 \mu \mathrm{m}$. BMSC, bone marrow mesenchymal stem cell; DPI, DPIYALSWSGMA; FITC, fluorescein isothiocyanate; LSP, LSPSAGAYIDWM.

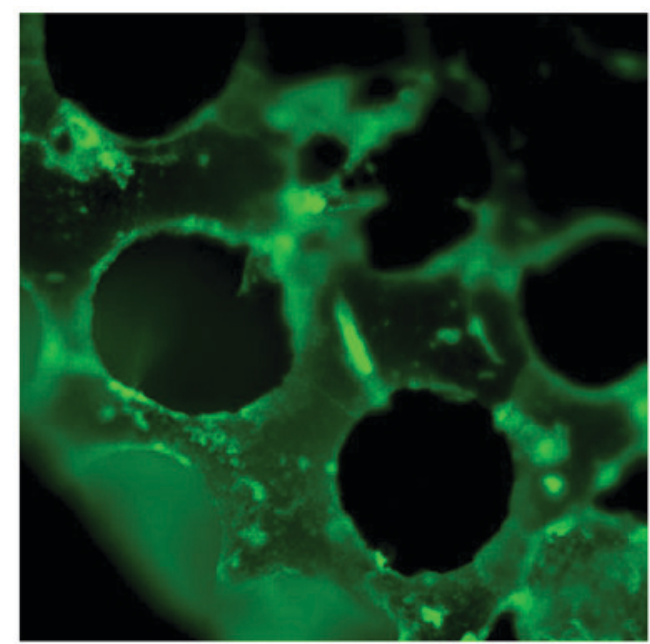

Figure 6. Successful synthesis of functional $\beta$-TCP scaffolds modified by DPI through an adsorption/freeze-drying strategy. FITC-labeled DPI peptide was adsorbed onto $\beta$-TCP through an adsorption/freeze-drying strategy to fabricate functional $\beta$-TCP-DPI scaffolds. Following adsorption, the $\beta$-TCP scaffolds exhibited a homogeneous green fluorescence. $\beta$-TCP, $\beta$-tricalcium phosphate; DPI, DPIYALSWSGMA; FITC, fluorescein isothiocyanate.

towards BMSCs. It was also demonstrated that the $\beta$-TCP-DPI scaffolds enhanced the adhesion and proliferation of BMSCs compared with pure $\beta$-TCP scaffolds.

$\beta$-TCP is commonly used in absorbable bioceramics (27). In tissue engineering, $\beta$-TCP is an ideal biomaterial for the repair of osteonecrosis or bone defects $(2,28)$. $\beta$-TCP has been extensively studied and applied as a bone repair and bone tissue engineering scaffold material (27). It is non-cytotoxic and has excellent biocompatibility and osteo-conductivity $(3,4)$. Stable associations between peptides and materials are essential for the construction of functional scaffolds (13). Methods of connecting peptide molecules to materials include covalent attachment, blending, co-polymerization, chemical and physical

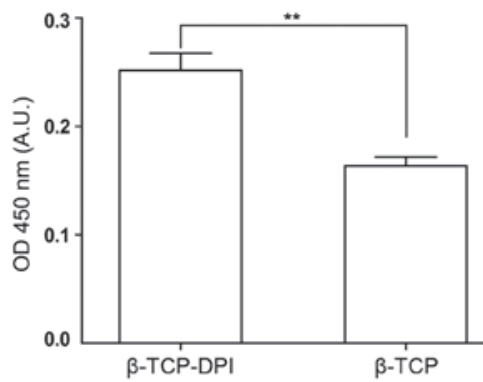

Figure 7. Adhesion of C57BL/6 mouse BMSCs to the functional $\beta$-TCP scaffolds is enhanced. Adhesion of BMSCs was measured by Cell Counting Kit- 8 assay $3 \mathrm{~h}$ after cell suspension was added to 96 -well plates containing $\beta$-TCP-DPI and pure $\beta$-TCP scaffolds. The OD value of the $\beta$-TCP-DPI scaffolds was significantly higher compared with that of the pure $\beta$-TCP scaffolds, which indicated that the number of adhesive cells in the $\beta$-TCP-DPI scaffold group was higher compared with the pure $\beta$-TCP scaffold group. Data are expressed as the mean \pm standard deviation; $n=3 ;{ }^{* *} \mathrm{P}<0.01$ vs. $\beta$-TCP. $\beta$-TCP, $\beta$-tricalcium phosphate; A.U., arbitrary units; BMSC, bone marrow mesenchymal stem cell; DPI, DPIYALSWSGMA; OD, optical density.

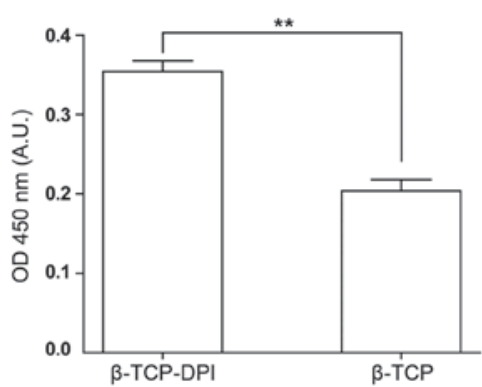

Figure 8. Proliferation of C57BL/6 mouse BMSCs is enhanced on $\beta$-TCP-DPI scaffolds. A Cell Counting Kit- 8 assay was used to measure the proliferation of BMSCs on $\beta$-TCP-DPI and pure $\beta$-TCP scaffolds. Following 3 days of incubation, the OD value of the $\beta$-TCP-DPI scaffolds was higher compared with that of the pure $\beta$-TCP scaffolds, which indicated that $\beta$-TCP modified by DPI may enhance BMSC proliferation compared with unmodified $\beta$-TCP. Data are expressed as the mean \pm standard deviation; $n=3 ;{ }^{* *} \mathrm{P}<0.01$ vs. $\beta$-TCP. $\beta$-TCP, $\beta$-tricalcium phosphate; A.U., arbitrary units; BMSC, bone marrow mesenchymal stem cell; DPI, DPIYALSWSGMA; OD, optical density. 
treatment (13). As an inorganic material, $\beta$-TCP has no innate biological stimulatory activity and lacks functional groups. Biomolecules are, therefore, difficult to conjugate to $\beta$-TCP (29). Amino acids, peptides and proteins can be adsorbed to inorganic materials (30-32). The mechanism of adsorption involves the formation of complexes between the carboxyl group and surface $\mathrm{Ca}^{2+}$, between free amino or guanidine groups and the phosphate group (10). Thus, an adsorption/freeze-drying strategy was used in the present study to modify $\beta$-TCP scaffolds with DPI $(10,16,33)$. In the process of synthesizing functional $\beta$-TCP, unadsorbed peptide should be rinsed sufficiently; otherwise non-adsorbed peptide molecules may dissolve in the cell culture medium and inhibit cell adhesion (12).

The present study aimed to investigate the ability of C57BL/6 mouse BMSCs to adhere and proliferate on $\beta$-TCP-DPI scaffolds. In the cell adhesion assay, the CCK- 8 assay was used to detect the number of adherent cells on the scaffolds. It was hypothesized that BMSCs would be recruited onto the $\beta$-TCP-DPI scaffolds more strongly and rapidly compared with the pure $\beta$-TCP scaffolds. Cell adhesion to substrates is time-dependent, and experimental adhesion time should be carefully considered (13). It usually assessed 1-4 h after cell seeding, and in the present study, $3 \mathrm{~h}$ was selected as the time point of verification. The results indicated that the adhesion of BMSCs to the functional $\beta$-TCP scaffolds was enhanced compared with the pure $\beta$-TCP scaffolds.

Numerous biomolecules have been reported to promote the proliferation of BMSCs $(6,34)$. Therefore, it was considered necessary to study the effect of the functional $\beta$-TCP scaffolds on proliferation. In cell adhesion assay, cell suspension was added into the wells and cells adhered to the scaffolds freely. In cell proliferation assay, cells were seeded onto the scaffolds restrictedly; cell suspension was carefully placed onto the center-top surface of the scaffolds to ensure that all cells adhered to the scaffolds. Therefore, the initial number of cells growing on scaffolds was fixed to accurately investigate the changes of cell proliferation. The result indicated that DPI-modified $\beta$-TCP scaffolds promoted the proliferation of BMSCs.

Additional studies are required to investigate the underlying mechanism of the affinity peptide towards BMSCs. The repair effect of the functional $\beta$-TCP-DPI scaffolds should also be further studied and evaluated in vivo.

In the present study, functional $\beta$-TCP scaffolds were successfully synthesized by adsorption of the BMSC affinity peptide, DPI, onto the surface of $\beta$-TCP using an adsorption/freeze-drying strategy. In vitro experiments demonstrated that the adhesion and proliferation of BMSCs on the functional $\beta$-TCP scaffolds was enhanced. The functional scaffold may be used as a potent biomaterial for MSC-based tissue engineering therapy.

\section{Acknowledgements}

The authors would like to thank Dr Nianping Zhang, Dr Tiantong Sun and Dr Li Qiao for revising the manuscript.

\section{Funding}

The present study was supported by The National Natural Science Foundation of China (grant no. 81271966).

\section{Availability of data and materials}

The data sets generated and analyzed during the current study are available from the corresponding author on reasonable request.

\section{Authors' contributions}

GW, ZM and SS designed the experiments. GW performed the experiments. GW, HX, YL and CW analyzed the data. GW wrote the manuscript. GW revised the manuscript. All authors reviewed the manuscript.

\section{Ethics approval and consent to participate}

Not applicable.

\section{Patient consent for publication}

Not applicable.

\section{Competing interests}

The authors declare they have no competing interests.

\section{References}

1. Mont MA, Cherian JJ, Sierra RJ, Jones LC and Lieberman JR: Nontraumatic osteonecrosis of the femoral head: Where do we stand today? A ten-year update. J Bone Joint Surg Am 97: 1604-1627, 2015.

2. Tatara AM and Mikos AG: Tissue engineering in orthopaedics. J Bone Joint Surg Am 98: 1132-1139, 2016.

3. Ke D, Dernell W, Bandyopadhyay A and Bose S: Doped tricalcium phosphate scaffolds by thermal decomposition of naphthalene: Mechanical properties and in vivo osteogenesis in a rabbit femur model. J Biomed Mater Res B Appl Biomater 103: 1549-1559, 2015.

4. LeGeros RZ: Properties of osteoconductive biomaterials: Calcium phosphates. Clin Orthop Relat Res: 81-98, 2002.

5. Pittenger MF, Mackay AM, Beck SC, Jaiswal RK, Douglas R, Mosca JD, Moorman MA, Simonetti DW, Craig S and Marshak DR: Multilineage potential of adult human mesenchymal stem cells. Science 284: 143-147, 1999.

6. Li Q, Xing D, Ma L and Gao C: Synthesis of E7 peptide-modified biodegradable polyester with the improving affinity to mesenchymal stem cells. Mater Sci Eng C Mater Biol Appl 73: 562-568, 2017.

7. Herrmann M, Verrier S and Alini M: Strategies to stimulate mobilization and homing of endogenous stem and progenitor cells for bone tissue repair. Front Bioeng Biotechnol 3: 79, 2015.

8. Shao ZX, Zhang X, Pi YB, Yin L, Li L, Chen H, Zhou C and Ao Y: Surface modification on polycaprolactone electrospun mesh and human decalcified bone scaffold with synovium-derived mesenchymal stem cells-affinity peptide for tissue engineering. J Biomed Mater Res A 103: 318-329, 2015.

9. Pi Y, Zhang X, Shi J, Zhu J, Chen W, Zhang C, Gao W, Zhou C and Ao Y: Targeted delivery of non-viral vectors to cartilage in vivo using a chondrocyte-homing peptide identified by phage display. Biomaterials 32: 6324-6332, 2011.

10. Qian JJ and Bhatnagar RS: Enhanced cell attachment to anorganic bone mineral in the presence of a synthetic peptide related to collagen. J Biomed Mater Res 31: 545-554, 1996.

11. Bhatnagar RS, Qian JJ and Gough CA: The role in cell binding of a beta-bend within the triple helical region in collagen alpha 1 (I) chain: Structural and biological evidence for conformational tautomerism on fiber surface. J Biomol Struct Dyn 14: 547-560, 1997.

12. Pierschbacher MD and Ruoslahti E: Cell attachment activity of fibronectin can be duplicated by small synthetic fragments of the molecule. Nature 309: 30-33, 1984. 
13. Hersel U, Dahmen $C$ and Kessler H: RGD modified polymers: Biomaterials for stimulated cell adhesion and beyond. Biomaterials 24: 4385-4415, 2003.

14. Zhang $\mathrm{H}$ and Hollister S: Comparison of bone marrow stromal cell behaviors on poly (caprolactone) with or without surface modification: Studies on cell adhesion, survival and proliferation. J Biomater Sci Polym Ed 20: 1975-1993, 2009.

15. Ramaraju H, Miller SJ and Kohn DH: Dual-functioning peptides discovered by phage display increase the magnitude and specificity of BMSC attachment to mineralized biomaterials Biomaterials 134: 1-12, 2017.

16. Bhatnagar RS, Qian JJ, Wedrychowska A, Sadeghi M, Wu YM and Smith N: Design of biomimetic habitats for tissue engineering with $\mathrm{P}-15$, a synthetic peptide analogue of collagen. Tissue Eng 5: 53-65, 1999.

17. Zhang HX, Zhang XP, Xiao GY, Hou Y, Cheng L, Si M, Wang SS, $\mathrm{Li} \mathrm{YH}$ and Nie L: In vitro and in vivo evaluation of calcium phosphate composite scaffolds containing BMP-VEGF loaded PLGA microspheres for the treatment of avascular necrosis of the femoral head. Mater Sci Eng C Mater Biol Appl 60: 298-307, 2016.

18. Langer $\mathrm{R}$ and Vacanti JP: Tissue engineering. Science 260 : 920-926, 1993

19. Maijenburg MW, van der Schoot CE and Voemans C: Mesenchymal stromal cell migration: Possibilities to improve cellular therapy. Stem Cells Dev 21: 19-29, 2012.

20. Caplan AI: New era of cell-based orthopedic therapies. Tissue Eng Part B Rev 15: 195-200, 2009.

21. Caplan AI: Why are MSCs therapeutic? New data: New insight. J Pathol 217: 318-324, 2009.

22. Zhang H, Lin CY and Hollister SJ: The interaction between bone marrow stromal cells and RGD-modified three-dimensional porous polycaprolactone scaffolds. Biomaterials 30: 4063-4069, 2009.

23. Shao Z, Zhang X, Pi Y, Wang X, Jia Z, Zhu J, Dai L, Chen W, Yin L, Chen $\mathrm{H}$, et al: Polycaprolactone electrospun mesh conjugated with an MSC affinity peptide for MSC homing in vivo. Biomaterials 33: 3375-3387, 2012.

24. Meng Q, Man Z, Dai L, Huang H, Zhang X, Hu X, Shao Z, Zhu J, Zhang J, Fu X, et al: A composite scaffold of MSC affinity peptide-modified demineralized bone matrix particles and chitosan hydrogel for cartilage regeneration. Sci Rep 5: 17802, 2015 .
25. Huang H, Zhang X, Hu X, Shao Z, Zhu J, Dai L, Man Z, Yuan L, Chen $\mathrm{H}$, Zhou C and Ao Y: A functional biphasic biomaterial homing mesenchymal stem cells for in vivo cartilage regeneration. Biomaterials 35: 9608-9619, 2014.

26. Man Z, Yin L, Shao Z, Zhang X, Hu X, Zhu J, Dai L, Huang H, Yuan L, Zhou C, et al: The effects of co-delivery of BMSC-affinity peptide and rhTGF- $\beta 1$ from coaxial electrospun scaffolds on chondrogenic differentiation. Biomaterials 35: 5250-5260, 2014.

27. Liu B and Lun DX: Current application of $\beta$-tricalcium phosphate composites in orthopaedics. Orthop Surg 4: 139-144, 2012.

28. Rh Owen G, Dard M and Larjava H: Hydoxyapatite/beta-tricalcium phosphate biphasic ceramics as regenerative material for the repair of complex bone defects. J Biomed Mater Res B Appl Biomater 106: 2493-2512, 2018.

29. Alvarez LM, Rivera JJ, Stockdale L, Saini S, Lee RT and Griffith LG: Tethering of epidermal growth factor (EGF) to beta tricalcium phosphate ( $\beta \mathrm{TCP})$ via fusion to a high affinity, multimeric $\beta$ TCP-binding peptide: Effects on human multipotent stromal cells/connective tissue progenitors. PLoS One 10: e0129600, 2015.

30. Moreno EC, Kresak M and Hay DI: Adsorption of molecules of biological interest onto hydroxyapatite. Calcif Tissue Int 36: 48-59, 1984.

31. Hay DI and Moreno EC: Differential adsorption and chemical affinities of proteins for apatitic surfaces. J Dent Res 58: 930-942, 1979.

32. Gorbunoff MJ and Timasheff SN: The interaction of proteins with hydroxyapatite. III. Mechanism. Anal Biochem 136: 440-445, 1984.

33. Yang XB, Bhatnagar RS, Li S and Oreffo RO: Biomimetic collagen scaffolds for human bone cell growth and differentiation. Tissue Eng 10: 1148-1159, 2004.

34. Parrish B, Breitenkamp RB and Emrick T: PEG- and peptide-grafted aliphatic polyesters by click chemistry. J Am Chem Soc 127: 7404-7410, 2005.

This work is licensed under a Creative Commons Attribution-NonCommercial-NoDerivatives 4.0 International (CC BY-NC-ND 4.0) License. 